\title{
A UNIVERSAL SNAKE-LIKE CONTINUUM ${ }^{1}$
}

RICHARD M. SCHORI

1. Introduction. By a snake-like continuum we mean a compact metric chainable continuum. In [2], Mioduszowski discusses snakelike continua and calls a pseudo-arc the universal snake-like continuum since, as proved in [2], every snake-like continuum is a continuous image of a pseudo-arc. This usage of "universal" is at variance with the homeomorphic imbedding usage of universal and thus the question arises: Is there a universal snake-like continuum $X$ in the sense that every snake-like continuum can be imbedded in $X$ ? In this paper we construct such a universal snake-like continuum. This construction is based on the well-known theorem that $X$ is snake-like if and only if $X$ is the limit of an inverse sequence of arcs.

2. The construction. Let $I$ denote the closed unit interval with the usual topology and let $C(I)$ be the space of all continuous functions from $I$ into $I$ with the topology of uniform convergence. $C(I)$ is a separable metric space and thus there exists a countable dense subset $\left\{u_{1}, u_{2}, \cdots\right\}$ of $C(I)$.

If $n$ is a positive integer, let $I_{n}$ denote $I$ and for each $n$ greater than one we construct a continuous function $g_{n}: I_{n} \rightarrow I_{n-1}$. The limit $U$ of the resulting inverse sequence $\left(I_{i}, g_{i}\right)$ will be a universal snake-like continuum.

Let $u_{0}: I \rightarrow I$ be the identity function and if $i$ is an integer, define $E(i)$ such that $E(i)=i / 2$ if $i$ is even, and $E(i)=(i-1) / 2$ if $i$ is odd. If $n \geqq 2$ and $i$ is an integer such that $0 \leqq i<2^{n-1}$, let

$$
g_{n}(x)=2^{2-n} E(i)+2^{1-n} u_{(n-1)[i \bmod 2]}\left(2^{n} x-2 i\right)
$$

for all $x$ in $\left[2 i 2^{-n},(2 i+1) 2^{-n}\right]$. If $0<i<2^{n-1}$, let

$$
\text { (**) } g_{n}(x)=\frac{g_{n}\left(2 i 2^{-n}\right)-g_{n}\left((2 i-1) 2^{-n}\right)}{2 i 2^{-n}-(2 i-1) 2^{-n}}\left(x-2 i 2^{-n}\right)+g_{n}\left(2 i 2^{-n}\right)
$$

for $x$ in $\left[(2 i-1) 2^{-n}, 2 i 2^{-n}\right]$, and suppose $g_{n}(x)=g_{n}\left(\left(2^{n}-1\right) 2^{-n}\right)$ for $x$ in $\left[\left(2^{n}-1\right) 2^{-n}, 1\right]$.

Thus, we have divided $I_{n}$ into $2^{n}$ equal subintervals and by $\left({ }^{*}\right)$ we have defined a continuous function $g_{n}$ on the odd subintervals. The

Presented to the Society, April 25, 1964; received by the editors August 7, 1964.

1 This paper is part of the author's doctoral dissertation at the University of Iowa, prepared under the supervision of Professor S. Armentrout while the author was supported on an NSF Cooperative Fellowship. 
endpoints of the even subintervals have been defined and thus, by $(* *)$, we make linear extensions of $g_{n}$ to the even subintervals. Hence, $g_{n}$ is a continuous function from $I_{n}$ into $I_{n-1}$.

Hence, for all $i$ greater than one we have defined a continuous function $g_{i}: I_{i} \rightarrow I_{i-1}$. Let $\left(I_{i}, g_{i}\right)$ be the corresponding inverse sequence of arcs and hence the limit space $U$ is a snake-like continuum.

TheOREM. U contains a homeomorphic copy of every snake-like continuum.

Proof. Let $X$ be a snake-like continuum and let $\left(I_{i}, f_{i}\right)$ be an inverse sequence of arcs whose limit is $X$. Theorem 3 of Morton Brown's paper [1] essentially says that if we approximate each of the functions $f_{i}$ close enough, then we still obtain $X$ as the limit. Since $\left\{u_{1}, u_{2}, \cdots\right\}$ is a dense subset of $C(I)$, the $u_{j}$ can be used to approximate the $f_{i}$ and thus, with the aid of [1], it follows that there exists an increasing sequence $\left(n_{2}, n_{3}, \cdots\right)$ of positive integers such that (1) $u_{r_{i}}$ approximates $f_{i}$ and (2) $X$ is homeomorphic to the limit $Y$ of the inverse sequence $\left(I_{i}, u_{n_{i}}\right)$. It is clear that there is a sequence $\left(n_{2}, n_{3}, \cdots\right)$ such that if $i \geqq 2$ then $n_{i}+1<n_{i+1}$.

We now show that there exists a subspace of $U$ homeomorphic to $Y$ and thus to $X$. Let $J_{1}$ denote the subinterval $[0,1 / 2]$ of $I_{1}$, let $n_{1}=0$, and $\sum_{j=2}^{1} 2^{-n_{j}}=0$. If $k=2,3, \cdots$, let $J_{k}$ be the subinterval

$$
\left[\sum_{j=2}^{k} 2^{-n_{j}}, \sum_{j=2}^{k} 2^{-n_{j}}+2^{-n_{k}-1}\right] \text { of } I_{n_{k}+1} \text {. }
$$

Also define $w_{k}: J_{k} \rightarrow I_{n_{k-1}+1}$ such that

$$
w_{k}=g_{n_{k-1}+2} \circ \cdots \circ g_{n_{k+1}} \mid J_{k} .
$$

For instance, if $n_{2}=1$ and $n_{3}=3$, then $J_{2}$ and $J_{3}$ are the respective subintervals $[1 / 2,3 / 4]$ and $[10 / 16,11 / 16]$ of $I_{2}$ and $I_{4}$. Thus, $w_{2}=g_{2} \mid J_{2}$ is the compressed $u_{1}$ and $w_{3}=g_{3} g_{4} \mid J_{3}$ is the compressed $u_{3}$ followed by the compressed identity.

$$
\text { LEMMA. } w_{k}(x)=\sum_{j=2}^{k-1} 2^{-n_{j}}+2^{-n_{k-1}-1} u_{n_{k}}\left(2^{n_{k}+1} x-2^{n_{k}+1} \sum_{j=2}^{k} 2^{-n_{j}}\right) \text {. }
$$

The proof of the lemma follows the proof of the theorem.

Thus we see that $w_{k}$ is essentially $u_{n_{k}}$ and furthermore, that $w_{k}\left[J_{k}\right] \subset J_{k-1}$. Hence, we have defined the inverse sequence $\left(J_{i}, w_{i}\right)$ and will prove that its limit, $W$, is homeomorphic to $Y$. Clearly $W$ is a subset of $U$. If $k=1,2, \cdots$, define the homeomorphism $q_{k}: J_{k}$ $\rightarrow I_{k}$ as follows:

$$
q_{k}(x)=2^{n_{k}+1} x-2^{n_{k+1}} \sum_{j=2}^{k} 2^{-n_{i}} .
$$


By straightforward calculations, we see that the diagram

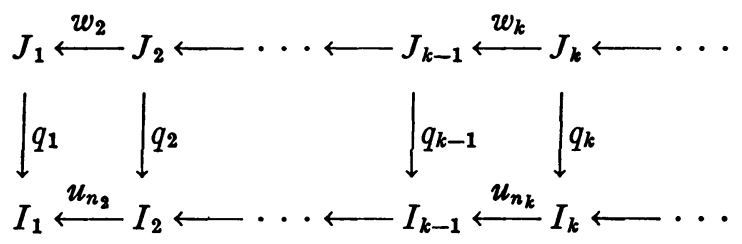

is commutative. That is, if $k=2,3, \cdots$, then $q_{k-1} w_{k}=u_{n_{k}} q_{k}$. Therefore we have a mapping $\left(q_{i}\right):\left(J_{i}, w_{i}\right) \rightarrow\left(I_{i}, u_{n_{i}}\right)$ which induces a homeomorphism $q: W \rightarrow Y$. Hence, we have a subspace $W$ of $U$ that is homeomorphic with the snake-like continuum $X$ and thus $U$ is a snake-like continuum that contains a homeomorphic copy of every snake-like continuum.

ProOF OF THE LEMMA. If $i=2^{n_{k}} \sum_{j=2}^{k} 2^{-n_{j}}=\left(\sum_{j=2}^{k-1} 2^{n_{k}-n_{j}}\right)+1$, then $i$ is an odd integer since $n_{k}-n_{j}>0$ where $j=1, \cdots, k-1$. Thus,

$$
J_{k}=\left[2 i 2^{-n_{k-1}},(2 i+1) 2^{-n_{k}-1}\right]
$$

and

$$
E(i)=\frac{1}{2} \sum_{j=2}^{k-1} 2^{n_{k}-n_{j}}=2^{n_{k}-1} \sum_{j=2}^{k-1} 2^{-n_{j}}
$$

and hence, by the definition of $g_{n_{k}+1}$,

$$
g_{n_{k}+1} \mid J_{k}=\sum_{j=2}^{k-1} 2^{-n_{j}}+2^{-n_{k}} u_{n_{k}}\left(2^{n_{k}+1} x-2^{n_{k}+1} \sum_{j=2}^{k} 2^{-n_{j}}\right) .
$$

With similar calculations including an inductive step, we show that if $0 \leqq p \leqq n_{k}-n_{k-1}-2$, then

$$
\begin{aligned}
g_{n_{k}-p} \circ \cdots \circ g_{n_{k}+1} & J_{k} \\
= & \sum_{j=2}^{k-1} 2^{-n_{j}}+2^{-n_{k}+p+1} u_{n_{k}}\left(2^{n_{k}+1} x-2^{n_{k}+1} \sum_{j=2}^{k} 2^{-n_{j}}\right) .
\end{aligned}
$$

In particular, it is true for $p=n_{k}-n_{k-1}-2$, and thus

$$
w_{k}(x)=\sum_{j=2}^{k-1} 2^{-n_{j}}+2^{-n_{k-1}-1} u_{n_{k}}\left(2^{n_{k}+1} x-2^{n_{k}+1} \sum_{j=2}^{k} 2^{-n_{j}}\right) \text {. }
$$

Corollary. If $X$ is any snake-like continuum, then $U$ contains $c$ mutually disjoint homeomorphic copies of $X$.

Proof. Just as there are $c$ distinct subsequences of the positive integers, there are $c$ ways of choosing the sequence $\left(n_{2}, n_{3}, \cdots\right)$ in 
the proof of Theorem 1 . The result follows since distinct subsequences yield disjoint subspaces of $W$.

Added in proof. With a slight modification $U$ can also be universal in the Mioduszowski continuous image sense. The point $(0,0, \cdots)$ of $U$ determined by the inverse limit representation is an endpoint and thus we can add a pseudo-arc $P$ such that $P \cup U$ is snake-like. If $X$ is any snake-like continuum, let $f$ map $P$ onto $X$ by [2] and extend $f$ to $P \cup U$ by collapsing $U$ to the appropriate point.

\title{
BIBLIOGRAPHY
}

1. Morton Brown, Some applications of an approximation theorem for inverse limits, Proc. Amer. Math. Soc. 11 (1960), 478-483.

2. J. Mioduszowski, A functional conception of snake-like continua, Fund. Math. 51 (1962), 179-189.

Louisiana State University

\section{A NOTE ON COUNTABLY PARACOMPACT SPACES AND METRIZATION}

\author{
C. E. AULL
}

As defined by Dowker [1], a topological space $(X, J)$ is called countably paracompact if every countable open cover of $X$ has an open locally finite refinement. It is known that every metrizable topological space is paracompact and hence countably paracompact.

It is proposed to show that in the usual metrization theorem for topological spaces $\left[3\right.$, p. 127], the $T_{3}$ condition may be replaced by the combination $T_{2}$ and countably paracompactness. We will need the following lemma.

LeммA. Every countably paracompact, first countable $T_{2}$ space is $T_{3}$.

Proof. Designate the topological space by $(X, J)$ as in Kelley [3]. Let $F$ be a proper closed set of $X$. For $x \notin F$, and $y \in F$ there exists disjoint open set $U_{x}^{(y)}$ and $U_{y}$ containing $x$ and $y$ respectively. There exists a countable base at $x,\left\{V_{k}\right\}, k=1,2, \cdots, n, \cdots$. Let $W_{k}=U\left\{U_{y}: U_{x}^{(y)} \supset V_{k}\right\} . W_{k} \cap V_{k}=\varnothing$ and $\left\{W_{k}\right\}$ forms a countable cover of $F$. Since $F$ is closed, $\left\{W_{k}\right\}$ may be replaced by an open locally finite refinement, $\left\{T_{a}: a \in A\right\}$ where $A$ is an index set.

Received by the editors December 28, 1964. 